\title{
Los estudios sobre calidad de vida y la perspectiva de la Psicología Positiva
}

Graciela Tonon`

\section{Resumen}

Las investigaciones sobre calidad de vida se desarrollaron a partir de la década del 70, si bien el antecedente del concepto data de los años 30 en Europa. En la actualidad, ha quedado establecido que la propuesta teórica de la calidad de vida alberga, como un paraguas, diferentes campos temáticos -la salud, el bienestar psicológico, el desarrollo económico, la vida social, las políticas públicas, la espiritualidad- comprendiendo dos dimensiones: la subjetiva y la objetiva. De esta manera, para estudiar y comprender la calidad de vida se requiere del aporte de diferentes disciplinas, convirtiéndose de esta manera en un concepto multidimensional.

La psicología ha contribuido a la investigación de la calidad de vida con conceptos tales como el de satisfacción vital y well-being subjetivo. La perspectiva de la Psicología Positiva con respecto a los estudios sobre calidad de vida, se conforma como mirada que permite conocer y poner énfasis en los atributos positivos de las personas, para enfrentar los cambios de la vida cotidiana, la crisis y la adversidad.

Palabras Clave: Calidad de vida - Psicología Positiva.

\section{Abstract}

The studies about quality of life have been developed since the 70 , even the origin of the concept has remained since the 30's. Nowadays, the theory about quality of life is like an umbrella, which includes different issues such as public policies, health, psychology, economy, social life, spirituality, etc. considering the

- Doctora en Ciencias Políticas. Chair South America and Board of Directors International Society for Quality of Life Studies (ISQOLS). Universidad de Palermo. E-mail: gracielatonon@hotmail.com 
objective and the subjective view. To study quality of life today, we need different disciplines so we can say that it is a multidimensional concept.

Psychology has contributed to the research in life quality with concepts such as life satisfaction and subjective well-being.

Positive Psychology related to the studies on life quality emphasizes people's positive attributes to deal with daily life changes, crisis and adversity.

Key Words: Quality of life - Positive Psychology. 


\section{Calidad de vida}

La calidad de vida es hoy concebida como un concepto multidimensional que comprende un número de dominios, considerados con diferente peso por cada persona, de acuerdo a la importancia que cada sujeto le asigna a cada uno de ellos.

Los orígenes del término se remontan a la década del 30 cuando el economista Pigou, pionero de la economía del bienestar, se refirió a cuantificar los servicios o costos sociales de las decisiones de gobierno para poder calcular un producto social neto. Pero fue el famoso texto de Campbell, Converse y Rodgers (1976) titulado The quality of american life: perceptions, evaluation and satisfactions, resultado de una investigación iniciada en 1971 por el Institute for Social Research (ISR) de la Universidad de Michigan, el que puso el tema sobre el tapete.

El trabajo de los autores se basó en considerar que las relaciones entre las condiciones denominadas objetivas y los estados psicológicos eran imperfectas y entonces, para conocer la experiencia de calidad de vida de una persona era necesario pedirle una descripción de cómo se sentía. El estudio consideró la satisfacción de la vida como un todo y se utilizó una escala con 17 dominios que conformaron un índice de calidad de vida. En dicho trabajo, el concepto de calidad de vida era equivalente al concepto de bienestar, y bienestar incluía: felicidad, satisfacción con la vida, afectos, estrés, ansiedad y competencias personales. Los autores señalaron que la evaluación que las personas hacían de su nivel de satisfacción, resultaba de la comparación que realizaban entre su medida o patrón de evaluación y su situación actual. Finalmente, señalaron que como se trataba de un primer estudio no recomendaban su utilización directa para la generación de políticas públicas, pero sí incentivaban a los equipos de investigación a continuar en esta línea de trabajo, proponiendo a los policy makers prestar atención a este nuevo tipo de estudios.

Dos décadas después, en 1995 se funda la International Society for Quality of Life Studies (ISQOLS) con el objetivo general de incentivar el desarrollo de estudios sobre la calidad de vida a nivel mundial; estimulando investigaciones interdisciplinarias en los campos de la psicología, la medicina, la política, lo social, y otras disciplinas dedicadas al desarrollo y el medio ambiente. En el año 1998 la ISQOLS elaboró el documento: Quality of Life Definition and Terminology: A Discussion Document from the International Society for Quality of Life Studies, trabajo coordinado por Cummins ${ }^{1}$, que muestra el resultado de la discusión entre los

1. Bob Cummins es el actual presidente de la ISQOLS y el Director del Australian Center on Quality of Life de la Deakin University, Australia y fundador del International Well-being group. 
expertos pioneros en el tema y que concluye con el reconocimiento de que la calidad de vida puede ser medida a nivel subjetivo y objetivo. En este documento, Vitterso (1998) planteó la necesidad de separar los componentes objetivos y subjetivos de la calidad de vida, considerando que el bienestar personal es un concepto más restringido que el de calidad de vida y es un vehículo para entender a los sujetos, sus sentimientos, motivaciones, pensamientos y acciones.

Hoy podemos decir que la calidad de vida es la percepción que tiene cada persona de su posición en la vida, en el contexto cultural y el sistema de valores en el cual vive, en relación con sus expectativas, intereses y logros. Resulta así un concepto complejo, que se relaciona con la salud física, la situación psicológica, el nivel de independencia, las relaciones sociales y las relaciones con el medio ambiente (WHOQOL group, 1995). Dado que las personas evalúan de manera diferente las circunstancias de su vida, dependiendo de sus expectativas, valores y experiencias previas, los estudios sobre calidad de vida reconocen la importancia de esas diferencias (Bramston, 2001).

La calidad de vida está condicionada por la estructura social, que puede ser considerada en términos de características demográficas, pautas culturales, características psicosociales de la comunidad, así como de las características de las instituciones, tanto en el ámbito estatal como en el privado, que desarrollan su accionar en ese contexto (Ferris, 2006); y es en este sentido que Cummins y Cahill (2000) consideraron que la calidad de vida se encuentra culturalmente definida.

Finalmente, y coincidiendo con Kreitler y Niv (2007) diremos que la calidad de vida es un concepto subjetivo, que refleja el punto de vista individual del bienestar y funcionamiento de cada sujeto. Es un constructo fenomenológico que provee una imagen de la situación sin explicar porqué, ni cómo surgió; es experiencial y evaluativo ya que presenta un juicio sin ningún intento de relacionarlo con variables objetivas; es dinámico, flexible y significativo para cada sujeto; y es multidimensional ya que no se basa solo en una medición global sino en dominios específicos identificados como constitutivos de la calidad de vida.

\section{La investigación en calidad de vida a nivel macro y la necesidad de considerar las especificidades regionales}

En el año 2001 Cummins et al., construyeron el Well-Being Index (WBI), instrumento que ha sido utilizado con mayor difusión internacional. El WBI está compuesto por dos escalas Likert del 0 al 10; la primera escala -Personal WellBeing (PWI)- mide el nivel de satisfacción de cada sujeto con su vida a nivel personal, en tanto la segunda escala, mide el nivel de satisfacción de cada sujeto con su vida en el país -National Well-Being-. El instrumento presenta un alto nivel de confiabilidad y hasta la fecha ha sido utilizado en 47 países de los diferentes 
continentes del planeta: Alemania, Argelia, Argentina, Australia, Bélgica, Bután, Canadá, China, Colombia, Croacia, Eslovaquia, España, Estados Unidos, Federación de las Indias Occidentales, Finlandia, Groenlandia, Holanda, Hungría, India, Inglaterra, Irán, Irlanda, Israel, Italia, Japón, Laos, Líbano, Malasia, Mauritania, México, Nueva Zelanda, Noruega, Pakistán, Filipinas, Polonia, Portugal, Rumania, Rusia, Ruanda, Singapur, Sudáfrica, Suiza, Tailandia, Taiwán, Turquía y Vietnam del Norte, a partir del trabajo desarrollado por los investigadores miembros del International Well-being Group ${ }^{2}$.

En Argentina, hicimos la traducción del instrumento (Tonon y Aguirre, 2002) y desde el año 2002 y hasta el año 2005 lo aplicamos a diferentes grupos poblacionales ${ }^{3}$.

En el año 2002, aplicamos el cuestionario a 500 sujetos de ambos sexos de entre 18 y 67 años de edad. En el año 2003, a 192 sujetos de ambos sexos de entre 18 y 67 años de edad. En el 2004, lo hicimos con 266 sujetos de ambos sexos de entre 15 y 18 años de edad, y en el 2005 con 289 sujetos de ambos sexos de entre 15 y 19 años de edad. Algunos de los resultados de estas investigaciones han sido presentados en las Conferencias Internacionales de la ISQOLS (Frankfurt 2003 y Filadelfia, 2004) y otros fueron publicados en el libro Juventud y protagonismo ciudadano (Tonon, 2006).

En el año 2004, Shah y Marks ${ }^{4}$ redactaron el manifiesto del well-being, documento en el cual se planteaba el interrogante acerca de lo que pueden hacer los gobiernos para mejorar la calidad de vida de la población. Los autores definían que la calidad de vida contiene dos dimensiones personales y una relativa al contexto social. Las personales corresponden a la satisfacción con la vida -que incluye satisfacción, placer y disfrute- y el desarrollo personal -que se define como estar comprometido con la vida, crecer en autonomía y sentir que la vida tiene sentido-. La dimensión social se refiere al sentimiento de pertenencia a la comunidad, a la actitud positiva hacía los demás, al sentimiento de compromiso hacia la comunidad y a colaborar con el desarrollo de la misma. Esto, porque consideran que el well-being no se reduce a una felicidad pasiva sino que incluye

2. La autora es Primary Researcher del International Well-being Group, Australian Center on Quality of Life, Deakin University, Australia

3. Investigaciones desarrolladas en el marco del International Well-being Group, ACQOL y el Programa de investigación en Calidad de Vida de la Facultad de Ciencias Sociales de la Universidad Nacional de Lomas de Zamora (la autora es la Coordinadora del Programa).

4. Nick Marks es el fundador de NEF, New Economic Foundation, UK, organización que obtuvo el Award for the Betterment of the Human Condition de la ISQOLS, 2007. 
el compromiso activo con la propia vida y con la de los demás. Los autores explicitan que uno de los objetivos de una sociedad democrática es promover la buena vida de los ciudadanos, logrando que las personas se sientan felices, sanas, capaces, es decir, que sientan un nivel alto de well-being.

En nuestro caso, nos encontramos en la actualidad trabajando en la producción de una nueva escala para medir el nivel de satisfacción de las personas con la calidad de vida en el país, que incorpore indicadores de relevancia regional. Esto es así porque, en la utilización tradicional de los sistemas de indicadores estadísticos en tanto representaciones de la realidad, el interés se ha centrado en lograr generalizaciones, y este proceso en la mayoría de los casos, ha presentado a los indicadores en forma aislada de su contexto histórico-social de desarrollo. En este sentido, Taylor (1993) ya señalaba que la universalidad de los indicadores no era un criterio permanente ya que los indicadores no podían ser evaluados fuera del contexto de su cultura. Siguiendo esta idea, se ha comenzado a generar una tendencia hacia la construcción de indicadores por región, más que a nivel internacional, de tal manera que el trabajo con indicadores permita generar una mirada que integre lo económico-político-psicosocial.

\section{Calidad de vida y Psicología Positiva}

La relación entre las propuestas teóricas de la calidad de vida y las de la Psicología Positiva ha quedado expresada tanto en la actividad de algunos académicos reconocidos en el campo temático -el caso de Ed Diener y Ruut Veenhoven, autores que forman parte del Board of Directors de la ISQOLS y de la Asociación Internacional de Psicología Positiva (IPPA)- así como en publicaciones especialmente dedicadas al tema, como el volumen 76, Número 1, del Social Indicators Research editado por la ISQOLS-Springer, de marzo del 2006, dedicado a la Psicología Positiva, que fue coordinado por Anastasia Efklides -School of Psychology- y Haralambos Tsorbatzoudis -School of Physical Education and Sport Sciences- de la Aristotle University of Tesalónica, Grecia.

Este volumen, presenta artículos que estudian los correlatos psicológicos con la calidad de vida, con énfasis en atributos humanos positivos, recursos personales, afectivos y espirituales que contribuyen a la calidad de vida, en poblaciones -jóvenes y adultos mayores- que se encuentran atravesando etapas de cambio, que pueden afectar sus recursos. Sus autores provienen de diferentes latitudes: Grecia, Israel y Estonia.

Efklides y Tsorbatzoudis (2006) concluyen diciendo, que este volumen ha puesto el énfasis en el estudio de los recursos humanos y los mecanismos psicológicos que intervienen entre los factores externos y el bienestar subjetivo. De esta manera desde el nivel descriptivo, muestra cómo interactúan las actitudes 
situacionales, contextuales y personales, particularmente en períodos de crisis, a fin de determinar el nivel de adaptación de cada persona y su calidad de vida.

Desde la Psicología Positiva, Boniwell (2006) señala que la misma opera en tres niveles, a los cuales denomina: nivel subjetivo, nivel individual y nivel grupal. El nivel subjetivo incluye el estudio de experiencias positivas como el bienestar, la satisfacción, la felicidad, entre otras. El nivel individual se dedica a la identificación de los elementos constitutivos de una "buena vida" y a las cualidades personales necesarias para ser una "buena persona". En el tercer nivel, la autora pone el énfasis en las virtudes cívicas, las responsabilidades sociales, la civilidad, la ética, las instituciones positivas y otros factores que podrían contribuir al desarrollo de la ciudadanía y la comunidad.

En el caso de la calidad de vida, y coincidiendo con Casas (1999), diremos que en la actualidad el concepto implica la participación de los ciudadanos en la evaluación de lo que les afecta, de tal manera que resulta portador de significación política, contribuyendo al estudio del bienestar de las personas, desde la perspectiva del bienestar físico y psicológico, relacionando las necesidades materiales con las socio-afectivas e integrando mediciones psicológicas y sociales de percepción y evaluación de las propias experiencias de los sujetos.

La psicología, en tanto campo disciplinar específico, ha contribuido a la investigación en calidad de vida con conceptos tales como el de satisfacción vital y well-being subjetivo. La perspectiva de la Psicología Positiva en los estudios sobre calidad de vida, se ha conformado como mirada que permite conocer los correlatos psicológicos de la calidad de vida, con énfasis en los atributos positivos de las personas, para enfrentar los cambios de la vida cotidiana, la crisis y la adversidad.

\section{Bibliografía}

Boniwell, I. (2006), Positive Psychology in a nutshell. London: PWBC.

Bramston, P. (2002), "Subjective quality of life: the affective dimension" En Gullone, E \& Cummins, R (Eds) Social Indicators Research Vol. 16. Kluwer Academic Publishers. The Netherlands. Pp. 47-62.

Campbell, A., Converse, P., Rodgers, E. (1976), The Quality of American Life: Perceptions, Evaluations and Satisfactions. New York: Russel Sage Foundation.

Casas, F. (1999), Calidad de vida y calidad humana. Revista Papeles del Psicólogo $N^{\circ} 74$. Madrid.

Cummins, R. (1998) (Ed.), Quality of Life Definition and Terminology: A Discussion Document from the International Society for Quality of Life Studies. Blacksburg, Virginia: The International Society for Quality-of-Life Studies. 
Cummins, R. \& Cahill J. (2000). Avances en la comprensión de la calidad de vida subjetiva. En Intervención psicosocial. Revista sobre igualdad y calidad de vida. Vol. 9 (2) Colegio Oficial de psicólogos de Madrid, Madrid, pp. 185-198.

Cummins, R. et al (2001), Well-being index.

Efklides, A. \& Tsorbatzoudis, H. Social Indicators Research vol. 76 N. 1. ISQOLS-Springer. The Nederland's.

Ferris, A. (2006), A theory of social structure and the quality of life. En Applied Research in Quality of Life Vol. 1. Springer. The Netherlands. Pp. 117-123.

Gullone, E \& Cummins, R. (2002) (Eds), Social Indicators Research Vol. 16. The Netherlands. Kluwer Academic Publishers.

Kreitler, S \& Nive, D. (2007), "Quality of life and coping in chronic pain patients" En: Kreitler, S, Beltuti, D., Lamberto, A. y Niv, D. (eds.) The handbook of chronic pain. Pp. 77-99. USA: Nova.

Power, M.; Bullinger, M \& WHOQOL Group. (2002), “The universality of quality of life: an empirical approach using the WHOQOL". Social Indicators Research Vol. 16. Kluwer Academic Publishers. The Netherlands. Pp. 129-149.

Shah, N \& Marks, N. (2004), A well-being manifesto for a flourishing society. London: Nef.

Taylor, Ch. (1993), El multiculturalismo y la política del reconocimiento. México: Fondo de Cultura Económica.

The International Wellbeing Group. Personal Wellbeing Index-Adult. Manual. The Australian Centre on Quality of Life, Deakin University. Melbourne. $2^{\text {nd }}$ Edition Junio 2005.

Tonon, G. \& Aguirre, V. (2002), Traducción del well-being index. International Well-being Group. Australian Center of Quality of Life. Deakin University. Australia. [Disponible en red en:] http://acqol.deakin.edu.au/inter_wellbeing/ Instrumento_Well-being_Argentina.pdf

Tonon, G. (2006) (comp.) Juventud y protagonismo ciudadano. Buenos Aires: Espacio Editorial.

Tonon, G. (2008) "Los estudios sobre Calidad de Vida en la Aldea Global, en América Latina y en Argentina. Revisión conceptual, avances y desafíos”. En: Lucero, P. (Ed.), Territorio y Calidad de Vida, una mirada desde la Geografía Local, Grupo de Estudios sobre Población y Territorio, Facultad de Humanidades, Universidad Nacional de Mar del Plata. EUDEM. Pp. 25-40. 
Tonon, G. (comp.), Desigualdades sociales y oportunidades ciudadanas. Buenos Aires: Espacio Editorial.

Veenhoven, R. (2000). “Why social policy needs subjective indicators?” En: Casas, F y Saurina, C. (edits). Proceedings of the Third Conference of the ISQOLS. Universidad de Girona. Pp. 807-817.

Vitterso, J. (1998). "Quality of life refers to both objective and subjective components”. En: Cummins, R. (Ed.), Quality of Life Definition and Terminology: A Discussion Document from the International Society for Quality of Life Studies. Blacksburg, Virginia: The International Society for Quality-of-Life Studies. 
